\title{
Topographie de l'Antiquité et du Moyen Âge (TOPAMA) : un outil Internet pour la cartographie SIG de données antiques et médiévales
}

\section{Thomas Lienhard}

\section{(2) OpenEdition}

\section{Journals}

Édition électronique

URL : http://journals.openedition.org/ifha/7388

DOI : 10.4000/ifha.7388

ISSN : 2198-8943

\section{Éditeur}

IFRA - Institut franco-allemand (sciences historiques et sociales)

\section{Édition imprimée}

Date de publication : 1 décembre 2013

ISSN : 2190-0078

\section{Référence électronique}

Thomas Lienhard, "Topographie de l'Antiquité et du Moyen Âge (TOPAMA) : un outil Internet pour la cartographie SIG de données antiques et médiévales », Revue de l'IFHA [En ligne], 5 | 2013, mis en ligne le 17 février 2014, consulté le 19 avril 2019. URL : http://journals.openedition.org/ifha/7388 ; DOI $10.4000 /$ ifha.7388

Ce document a été généré automatiquement le 19 avril 2019.

(CIFHA 


\title{
Topographie de l'Antiquité et du Moyen Âge (TOPAMA) : un outil Internet pour la cartographie SIG de données antiques et médiévales
}

\author{
Thomas Lienhard
}

Au printemps 2012 a vu le jour, à l'initiative de l'université de Paris-1 Panthéon-Sorbonne et de l'université Goethe de Francfort-sur-le-Main, un projet qui consistait à fournir aux internautes des instruments de cartographie historique répondant à trois objectifs : on voulait permettre à l'utilisateur de concevoir lui-même des cartes avec une qualité suffisante pour une publication, de superposer les représentations cartographiques de données historiques très diverses à des fins de comparaison, et enfin d'analyser des données de géographie historique avec les outils de la géomatique. Centré sur l'espace européen durant l'Antiquité chrétienne et le Moyen Âge, ce projet s'adresse à tous les enseignants et/ou chercheurs concernés par ce champ, mais peut intéresser plus spécifiquement les lecteurs de la présente revue par l'aspect franco-allemand qu'il comporte à un triple titre. D'une part, en effet, les données couvertes par le projet portent sur des espaces qui sont au moins franco-allemands, et on espère favoriser ainsi le dialogue entre les chercheurs de ces deux pays qui, pour des raisons institutionnelles, s'ignorent parfois mutuellement (ou sont contraints de le faire par les autorités qui les financent), cela alors que les phénomènes antiques ou médiévaux qu'ils étudient se rient évidemment de la frontière rhénane. D'autre part, la liste des partenaires associés au projet montre l'intérêt qu'une telle initiative peut susciter dans l'Université tant allemande que française: si les chercheurs impliqués sont trop nombreux pour être énumérés ici à titre individuel, signalons simplement que le programme bénéficie du partenariat ou du soutien de l'université Paris Ouest Nanterre La Défense (porteuse du projet au même titre que les deux universités fondatrices citées plus haut) ${ }^{1}$, de l'université de Strasbourg et notamment du projet ArkeoGIS'2 de l'université de HauteAlsace (qui produit en particulier l'Atlas Historique de l'Alsace ${ }^{3}$ ), du Ministère de la Culture et de la Communication avec la participation de plusieurs DRAC ${ }^{4}$ du CNRS et 
notamment du projet «Chartae Burgundiae Medii Aevi », de la Très Grande Infrastructure de Recherche Huma-Num (issue de la fusion entre Adonis et Corpus-IR) ${ }^{6}$, du projet de l'ERC «Signs and States " ${ }^{7}$ du Bundesministerium für Bildung und Forschung et spécialement du projet DARIAH-DE ${ }^{8}$, du Land de Bavière ${ }^{9}$, des Monumenta Germaniae Historica ${ }^{10}$, de la Germania Sacra ${ }^{11}$, du Deutsches Archäologisches Institut ${ }^{12}$, de l'Institut Historique Allemand de Paris $^{13}$ et de l'Institut Français d'Histoire en Allemagne ${ }^{14}$. Enfin, le programme est franco-allemand jusque dans le financement qu'il espère obtenir, puisqu'il a été soumis à l'Agence Nationale de la Recherche et à la Deutsche Forschungsgemeinschaft dans le cadre d'un appel à projets franco-allemand en sciences humaines et sociales. À l'heure de rédiger ces lignes, il est en cours d'évaluation par ces deux instances, le verdict étant attendu pour l'automne 2013: le lecteur comprendra donc qu'il est encore trop tôt pour présenter les moyens d'action financiers dont on espère disposer, mais on peut signaler dès à présent que même dans le cas d'un refus opposé par ces financeurs potentiels, des résultats tangibles verront le jour étant donné l'intérêt suscité par ce projet auprès des partenaires que l'on vient d'énumérer.

Plus précisément, de quoi s'agit-il? Les types d'objets à cartographier sont de trois ordres. En premier lieu seront pris en compte, à l'échelle de l'Europe occidentale, les données pour lesquelles les sources écrites antiques et médiévales permettent de construire des séries : seront traités notamment les évêchés (localisés sur la base de leur cathédrale) et les palais, ainsi qu'une sélection de monastères. En deuxième lieu, on intégrera dans le corpus les données archéologiques; pour ce point, le pragmatisme a exigé de réduire la focale, dans un premier temps, sur un espace plus restreint que l'Europe entière, en l'occurrence sur le grand Est français et le Sud-Ouest de l'Allemagne. Cette sélection permettait d'envisager un secteur qui est transfrontalier à la fois au regard des frontières actuelles et de celles de l'Antiquité, et donc de favoriser une approche comparative. En troisième lieu enfin, on souhaite cartographier des données lexicales: il s'agit là d'identifier le lieu de rédaction des nombreux textes antiques et médiévaux disponibles aujourd'hui au format numérisé, pour permettre des requêtes qui cartographieront chaque terme contenu dans ces sources (pour prendre un exemple parmi d'autres, on pourra ainsi déterminer où fut employé le terme de servus au IX ${ }^{\mathrm{e}}$ siècle, etc.).

3 Pour ces divers types de données, on offrira à l'utilisateur des outils à la fois de visualisation, d'analyse sommaire et de téléchargement dans un format aussi ouvert que possible. Ainsi, le site du projet permettra d'abord de voir la localisation des objets sur une carte d'Europe. Le mode de représentation choisi, un Système Géographique d'Information (SIG), présente l'avantage de permettre des changements d'échelle importants tout en préservant la légèreté des fichiers et, partant, la fluidité de la navigation : pour certaines données, on pourra ainsi passer en un seul clic de l'échelle européenne à celle d'un pâté de maisons, ce qui facilitera la coopération entre historiens et archéologues. Ce même système permet également de superposer des données très diverses, issues à la fois des informations géographiques actuelles et du travail des historiens concernant des époques reculées : on pourra ainsi sonder des causalités qui n'étaient pas évidentes auparavant, telles que l'éventuelle coïncidence entre la localisation des palais et celle des gisements de sel, ou encore entre celle des monastères et de telle ou telle pratique linguistique. L'ensemble des données sera daté, ce qui permettra à l'utilisateur de faire défiler facilement sur son écran l'évolution chronologique des objets historiques qui l'intéressent. Par ailleurs, le site Internet du 
projet offrira des outils d'analyse géomatique, permettant ainsi de produire des calculs de densité, de distance moyenne entre deux types d'objets, de difficulté des trajets en fonction du relief, etc.

4 Cela dit, quiconque s'est risqué à la géographie historique sait qu'aucun résultat trouvé en ligne ne peut permettre au chercheur de produire exactement la carte qu'il souhaite, ni de mener une analyse géographique personnalisée à propos d'un dossier historique. C'est la raison pour laquelle il est prévu de proposer, autant que possible, des données au format ouvert, de manière à ce que l'utilisateur puisse les télécharger et les manipuler aisément. Ce choix est l'une des principales caractéristiques qui distinguent le projet TOPAMA et d'autres initiatives qui se consacrent à des objets similaires. Il répond à des impératifs éthiques (de quel droit, après tout, verrouillerait-on des résultats qui ont été obtenus par des chercheurs financés par des fonds publics?), mais aussi à des considérations techniques concernant les usages que l'on veut offrir à la communauté scientifique: or l'objectif, en l'occurrence, ne consiste pas seulement à présenter des cartes toutes faites, mais également à proposer une base de données que l'enseignantchercheur pourra s'approprier pour ses propres travaux scientifiques ou ses projets d'enseignement. Dans leur majorité, les données du projet seront donc téléchargeables, gratuites et proposées dans des formats compatibles avec les logiciels libres disponibles sur la Toile ${ }^{15}$. Il est vrai que, dans certains cas, des contraintes d'ordre légal s'opposent inévitablement à une ouverture complète: dans le cas des données archéologiques en particulier, et notamment pour les sites aristocratiques ruraux, la législation allemande comme son homologue française interdisent une cartographie trop précise pour ne pas faciliter le travail des pilleurs. Cette considération, couplée avec l'imprécision notoire des sources antiques et médiévales en termes de localisation, empêche les concepteurs du projet de fournir l'intégralité de leurs résultats dans un format ouvert, ou les contraint à accepter un certain degré d'imprécision dans les localisations affichées.

D'où proviendront les données proposées ? Il faut ici distinguer entre deux cas de figure. Pour certains types d'objets, les membres du projet se livreront eux-mêmes à un travail de recollement, consultant les grandes collections documentaires (pour prendre un exemple parmi bien d'autres, citons la Carte archéologique de la Gaule) ou les sources antiques ou médiévales, pour en extraire et en adapter les résultats. Dans d'autres cas, le projet consistera à mettre en commun des bases de données qui existent déjà à l'heure actuelle, mais de manière dispersée. Précisons que dans cette seconde configuration, il s'agira d'une structure fédérale et non d'une fusion : chaque institution partenaire restera propriétaire des données qu'elle fournira au projet central, et libre de décider quel sera le détail des données qu'elle compte proposer, avec quel degré de précision elle le fera, durant combien de temps etc. Le site central constituera donc essentiellement une fenêtre commune pour des bases qui, concrètement, resteront hébergées et gérées sur des serveurs multiples. Cet aspect fédéral, qui constitue une autre caractéristique du projet TOPAMA, répond à la fois au souhait d'apaiser les craintes légitimes des institutions partenaires face à ce qui pourrait être perçu comme une dépossession intellectuelle, et, là encore, à des considérations d'ordre technique : non seulement il permet de réduire le volume des données conservées sur le site central, donc d'améliorer la fluidité de la navigation, mais il dispense également d'un lourd travail d'homogénéisation entre diverses bases de données dont la structure est très différente. Ainsi, le site hébergera notamment un outil permettant de "traduire » les métadonnées des différentes bases pour rendre celles-ci compatibles sur un même écran, sans que les 
institutions partenaires doivent modifier la structure originale de leurs données pour insérer celles-ci dans le projet central.

6 Par ces outils techniques, on espère pouvoir rajeunir des problématiques parfois anciennes de géographie historique. L'une d'entre elles pourrait désormais être considérée comme un pont aux ânes vu l'abondance de la production historiographique qu'elle a engendrée, si seulement elle avait reçu des réponses satisfaisantes : il s'agit des influences spatiales de l'Empire romain. Pour contribuer à ce chantier, le projet TOPAMA, et notamment sa composante archéologique, permettra de comparer les espaces qui étaient romains et ceux qui ne l'étaient pas, ou encore, pour un même secteur géographique, l'époque romaine et celle qui ne l'était plus : on obtiendra ainsi un élément d'analyse parmi d'autres pour comprendre les aspects spatiaux de l'influence romaine, et les motifs des évolutions ultérieures. Pour toute la période envisagée, on pourra également envisager les logiques territoriales : dans la définition des diocèses ou dans les partages politiques antiques et médiévaux, raisonnait-on en termes économiques de manière à ce que chaque entité soit viable, en termes culturels pour que chaque unité soit plus ou moins homogène, $y$ avait-il seulement une telle logique spatiale ou uniquement le hasard et le droit du plus fort? Pour toutes ces questions concernant les logiques spatiales européennes, que les historiens et archéologues français et allemands abordent en ordre dispersé voire en croisant le fer entre eux ${ }^{16}$, le projet TOPAMA s'efforcera d'apporter des éléments de réponse sur une période relativement longue.

7 À l'heure de rédiger ces lignes, le projet peut être consulté, sous une forme embryonnaire, sur le portail de Ménestrel ${ }^{17}:$ c'est là, en particulier, que l'on trouvera les données déjà disponibles, un mode d'emploi pour utiliser celles-ci, et le détail des développements ultérieurs.

\section{NOTES}

1. http://www.u-paris10.fr

2. http://arkeogis.org

3. http://www.atlas.historique.alsace.uha.fr

4. En particulier celle de Lorraine: http://www.culturecommunication.gouv.fr/Regions/DracLorraine

5. http://www.artehis-cnrs.fr/CBMA-Chartae-Burgundiae-Medii-Aevi,964

6. http://www.huma-num.fr

7. Il s'agit notamment de la Plateforme internet pour l'Analyse Linguistique de textes Médiévaux (http://lamop-vs3.univ-paris1.fr/PALM).

8. https://de.dariah.eu

9. http://www.bayern.de

10. http://www.mgh.de, en particulier http://www.dmgh.de pour les ressources textuelles électroniques.

11. http://adw-goe.de/forschung/forschungsprojekte-akademienprogramm/germania-sacra

12. http://www.dainst.org

13. http://www.dhi-paris.fr 
14. http://www.ifha.fr

15. L'utilisateur pourra ainsi télécharger des fichiers Shape, et obtenir les données sources sous forme de tableaux Excel. Ces deux formats ne sont certes pas libres, au sens juridique du terme, mais ils peuvent être importés dans des logiciels qui, eux, le sont.

16. On trouvera un bon aperçu de ces diverses approches dans deux publications soutenues par l'IFHA : Construction de l'espace au Moyen Âge : pratiques et représentations. Actes du $37^{e}$ colloque de la Société des Médiévistes de l'Enseignement Supérieur Public tenu à Mulhouse les 2, 3 et 4 juin 2006, Paris : Publications de la Sorbonne, 2007 ; plus récemment, Marion Picker, Véronique Maleval et Florent Gabaude (dir.), Die Zukunft der Kartographie. Neue und nicht so neue epistemologische Krisen, Bielefeld : transcript, 2013, et notamment l'article de Jens Schneider, « Raum und Grenze : Vergleichende Überlegungen zur Entwicklung im mittelalterlichen Reich », p. 177-197.

17. http://www.menestrel.fr/spip.php ?rubrique1621

\section{AUTEUR}

\section{THOMAS LIENHARD}

Université de Paris 1 Panthéon-Sorbonne 